\title{
Medical Image of the Week: MAC Infection
}
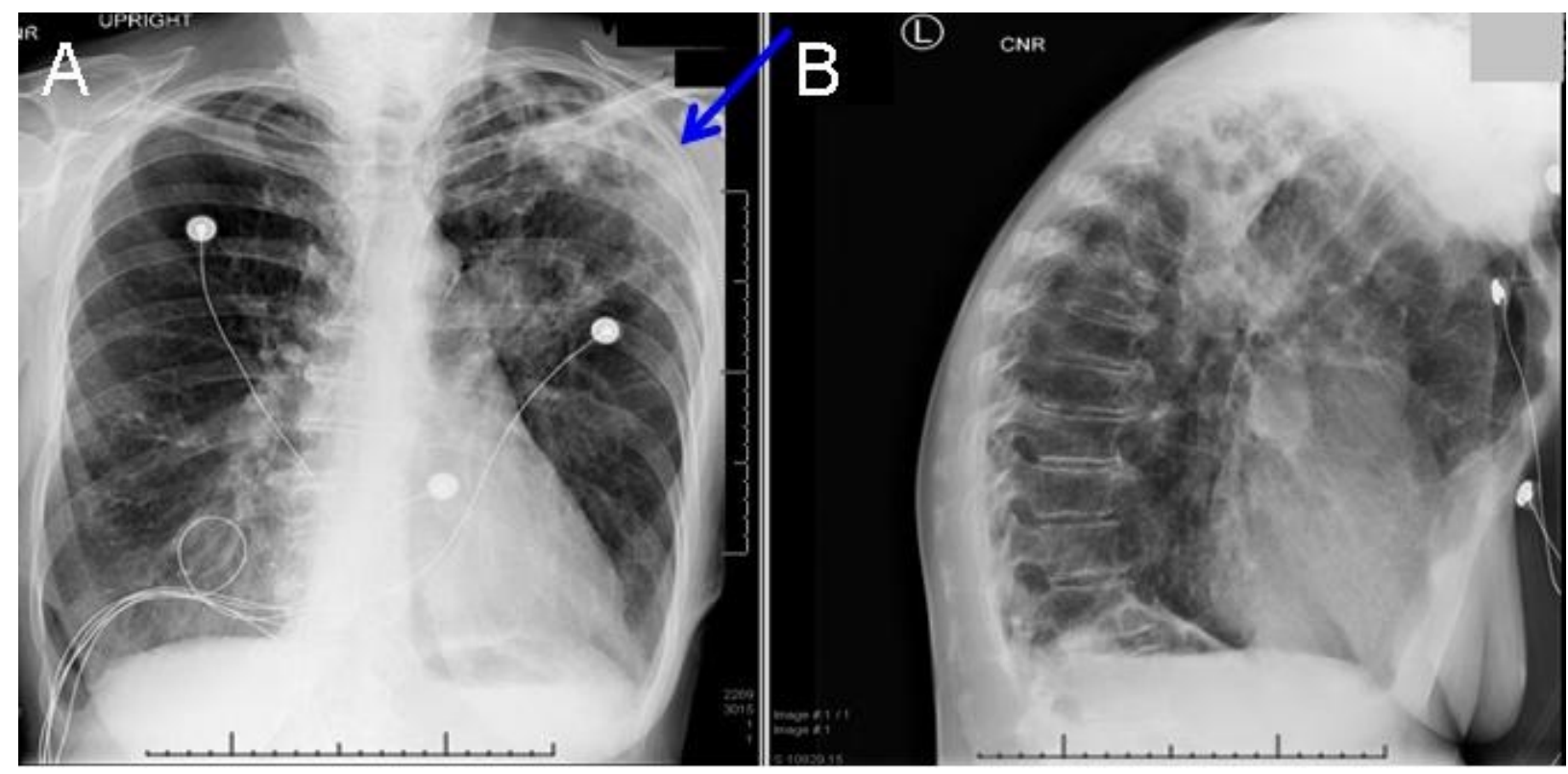

Figure 1. PA and lateral chest radiograph demonstrating left upper lobe air space disease with possible cavity (blue arrow).
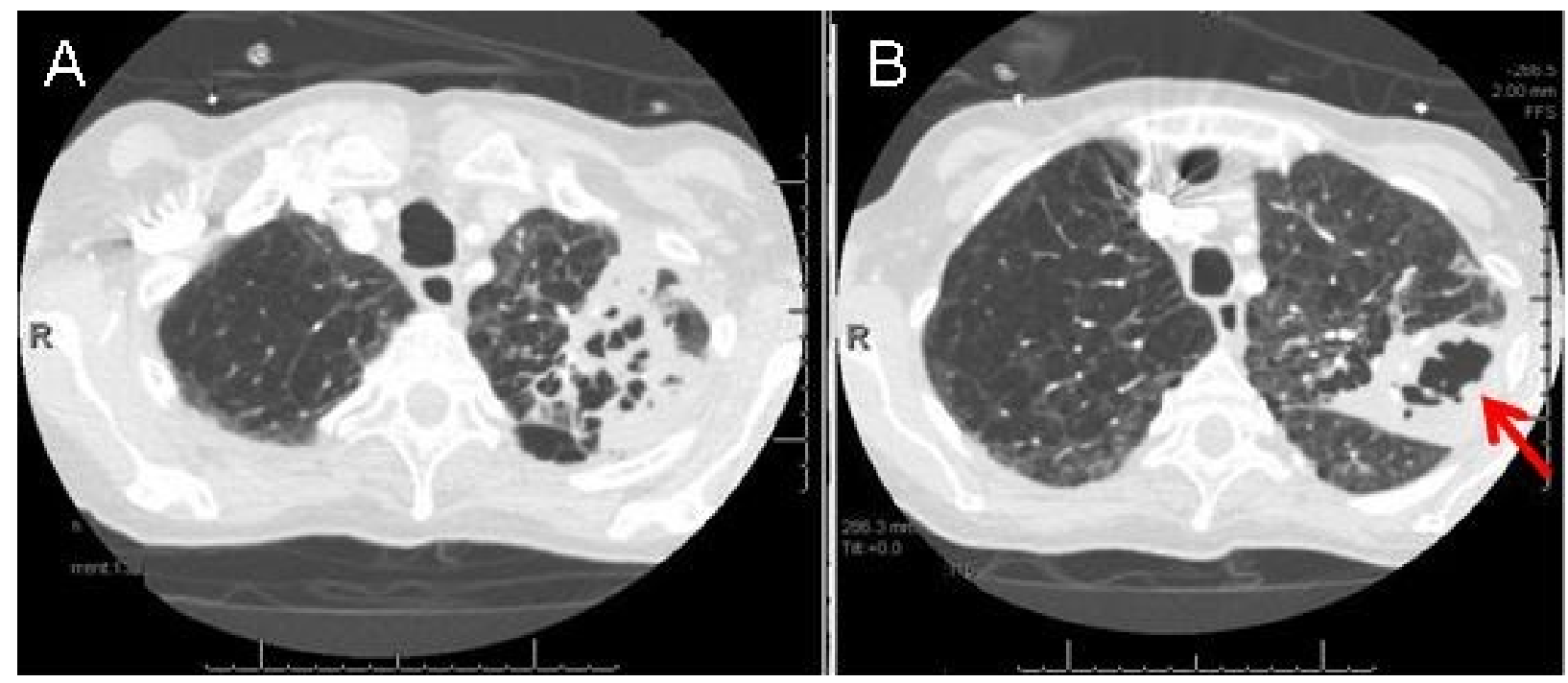

Figure 2. Chest CT (axial image) demonstrating extensive LUL cavitary necrotizing pneumonia (red arrow).

A 61-year-old woman with history of severe COPD (FEV1 1.07L, 40\%) complicated by chronic hypoxemic, hypercarbic respiratory failure, ongoing tobacco abuse, and allergic phenotype. Over the past month or so, she had developed progressively worsening 
dyspnea on exertion, fatigue, poor appetite, and weight loss. She denied fevers, chills, and night sweats. Thoracic CT did show LUL cavitary lesion and RLL sub segmental tiny pulmonary embolus.

A PA and lateral chest radiograph was performed and revealed extensive areas of patchy airspace opacity in the left upper lobe. Lucent foci are noted within the patchy opacities of concern for potential cavitation (Figure 1). CT chest was performed and showed extensive cavitary, necrotizing left upper lobe pneumonia, Centrilobular and paraseptal emphysema. (Figure 2). Sputum AFB was positive for acid fast bacilli, culture was positive for Mycobacterium avium complex (MAC), and she was started on treatment.

The term Mycobacterium avium complex (MAC) encompasses several species including $M$. avium and $M$. intracellulare. These organisms are genetically similar and generally not differentiated in the clinical microbiology laboratory. Among nontuberculosis mycobacterium, MAC is the most common cause of pulmonary disease worldwide. It is generally felt that these organisms are acquired from the environment. Mounting evidence suggests that municipal water sources may be an important source for MAC lung infections (1). Unlike $M$. tuberculosis, there are no convincing data demonstrating human-to-human or animal-to-human transmission of MAC.

Four major clinical presentations have been prescribed:

- Disease in those with known underlying lung disease, primarily white, middleaged, or elderly men, often alcoholics and/or smokers with underlying chronic obstructive pulmonary disease.

- Disease in those without known underlying lung disease predominantly in nonsmoking women over age 50 who have interstitial patterns on chest radiography.

- One report noted an unexpectedly high frequency (78 of 244 patients) of MAC pulmonary infections presenting as solitary pulmonary nodules, which resembled lung cancer (2).

- MAC exposure in immunocompetent hosts without underlying lung disease has been linked to the development of hypersensitivity pneumonitis, particularly following hot tub use.

The American Thoracic Society and Infectious Disease Society of America's diagnostic criteria for nontuberculosis mycobacterial pulmonary infections include both imaging studies consistent with pulmonary disease and at least two separate expectorated sputum samples isolation of mycobacteria or isolated from at least one bronchial wash in a symptomatic patient.

The recommendation is to start a combination of two to four drugs (as tolerated) for treatment of MAC pulmonary infection in HIV-negative patients. treatment for MAC until sputum cultures are consecutively negative for at least one year. 
The ATS/IDSA guidelines recommend a combination of clarithromycin (1000 mg three times per week) or azithromycin (500 mg three times per week) $\boldsymbol{P L U S}$ rifampin (600 mg three times per week) or rifabutin (300 mg three times per week) $\underline{P L U S}$ ethambutol (25 $\mathrm{mg} / \mathrm{kg}$ three times per week).

For patients with fibrocavitary MAC lung disease or severe nodular or bronchiectatic disease, the ATS/IDSA guidelines recommend same therapy plus streptomycin or amikacin (both 10 to $15 \mathrm{mg} / \mathrm{kg}$ three times per week) as a fourth agent for the first eight weeks. Patients receiving MAC treatment should have monthly monitoring for drug toxicity and sputum cultures.

Muna Omar, MD and Cristine Berry, MD Pulmonary, Critical Care, Sleep and Allergy Medicine Banner University Medical Center-Tucson Tucson, AZ USA

\section{References}

1. Mullis SN, Falkinham JO 3rd. Adherence and biofilm formation of Mycobacterium avium, Mycobacterium intracellulare and Mycobacterium abscessus to household plumbing materials. J Appl Microbiol. 2013 Sep;115(3):908-14. [CrossRef] [PubMed]

2. Teirstein AS, Damsker B, Kirschner PA, Krellenstein DJ, Robinson B, Chuang MT. Pulmonary infection with Mycobacterium avium-intracellulare: diagnosis, clinical patterns, treatment. Mt Sinai J Med. 1990 Sep;57(4):209-15. [PubMed]

3. An official ATS/IDSA statement: diagnosis, treatment, and prevention of nontuberculous mycobacterial diseases. Griffith DE, Aksamit T, Brown-Elliott BA, Catanzaro A, Daley C, Gordin F, Holland SM, Horsburgh R, Huitt G, lademarco MF, Iseman M, Olivier K, Ruoss S, von Reyn CF, Wallace RJ Jr, Winthrop K; ATS Mycobacterial Diseases Subcommittee; American Thoracic Society; Infectious Disease Society of America. Am J Respir Crit Care Med. 2007 Feb 15;175(4):367416. [CrossRef] [PubMed] 